\title{
Analysis of the efficiency of the linearization techniques for solving multi-objective linear fractional programming problems by goal programming
}

\author{
Tunjo Perićc ${ }^{1, \dagger}, Z_{\text {Zoran }}$ Babić $^{2}$ and Maid Omerović ${ }^{3}$ \\ ${ }^{1}$ Department of Mathematics, Faculty of Economics and Business, University of Zagreb, \\ J. F. Kennedy 6, 10000 Zagreb, Croatia \\ E-mail: 〈tperic@efzg.hr〉 \\ ${ }^{2}$ Department of Quantitative Methods, Faculty of Economics, University of Split, 21000 \\ Split, Croatia \\ E-mail:〈babic@efst.hr〉 \\ ${ }^{3}$ Faculty for Education, University of Travnik, 72270 Travnik, Bosnia and Herzegovina \\ E-mail:〈maid.omerovic@gmail.com 〉
}

\begin{abstract}
This paper presents and analyzes the applicability of three linearization techniques used for solving multi-objective linear fractional programming problems using the goal programming method. The three linearization techniques are: (1) Taylor's polynomial linearization approximation, (2) the method of variable change, and (3) a modification of the method of variable change proposed in [20]. All three linearization techniques are presented and analyzed in two variants: (a) using the optimal value of the objective functions as the decision makers' aspirations, and (b) the decision makers' aspirations are given by the decision makers. As the criteria for the analysis we use the efficiency of the obtained solutions and the difficulties the analyst comes upon in preparing the linearization models. To analyze the applicability of the linearization techniques incorporated in the linear goal programming method we use an example of a financial structure optimization problem.
\end{abstract}

Keywords: linear fractional programming model, linearization techniques, goal programming

Received: October 24, 2016; accepted: March 30, 2017; available online: March 31, 2017

DOI: $10.17535 /$ crorr.2017.0016

\section{Introduction}

There are many business and economics problems that can be presented by multiobjective linear fractional programming (MOLFP) models. A MOLFP model

\footnotetext{
$\dagger$ Corresponding author 
contains $k(k \geq 2)$ linear fractional objective functions that should be optimized (maximized or minimized) on the set $\mathbf{S}$ consisting of $m$ linear constraints. Therefore, what is to be found is an efficient preferred solution on the given set, which is a convex polyhedron.

The problem of linear fractional programming with one objective function was extensively researched in the second half of the twentieth century and efficient methods were developed for solving such problems ([3], [4]). Solving multiobjective linear fractional programming models is limited to a small number of inadequately effective multi-objective programming methods ([2], [7], [8], [9], [11], [12], [14], [16], [17], [18], [21], [22]).

The most effective method for solving multi-objective linear fractional programming models is the linear goal programming method based on the simplex algorithm. However, the use of linear goal programming for solving those problems requires linearization of the linear fractional objective functions.

For the linearization of the linear fractional objective functions we can use different methods which do not have the same efficiency. Here we present three linearization methods: 1) Taylor's polynomial linearization approximation [22], 2) the method of variable change ([9], [11], [12], [18]), and 3) a modification of the method of variable change proposed in [20].

The analysis of the linearization approaches will be done by solving a financial structure optimization problem with respect to efficiency of the obtained results and the difficulties the analysts come upon in preparing the model. As a result, we will obtain the most appropriate linearization method for solving such problems by using goal programming methods.

The rest of the paper is organized as follows: in the Section 2, we introduce the MOLFP model, the linear goal programming method and three linearization techniques for solving MOLFP problems by linear goal programming. In the Section 3, we solve a financial structure optimization problem by using presented linearization techniques and the linear goal programming method and analyze the applicability of the presented methods. In the Section 4, we give conclusions about the applicability of the linearization techniques and recommendations for the future research. 


\section{Goal programming methodology for solving MOLFP problems}

\subsection{MOLFP model}

A MOLFP model can be written as

$$
\max _{\mathbf{x} \in \mathbf{S}} Z=\left\{z_{1}, z_{2}, \ldots, z_{K}\right\}
$$

where $\mathbf{S}=\left\{\begin{array}{l}\mathbf{x}=\left[x_{1}, x_{2}, \ldots, x_{n}\right]^{T}: \\ A \mathbf{x} * \mathbf{b}, \mathbf{x} \geq \mathbf{0}\end{array}\right\}, \quad z_{k}=\frac{\sum_{j=1}^{n} c_{k j} x_{j}+c_{0}^{k}}{\sum_{j=1}^{n} d_{k j} x_{j}+d_{0}^{k}}=\frac{\mathbf{c}_{k} \mathbf{x}+c_{0}^{k}}{\mathbf{d}_{k} \mathbf{x}+d_{0}^{k}}$,

$k=1,2, \ldots, K$, are linear fractional functions, $A$ is an $(m, n)$ matrix, $\mathbf{x}$ is an $n-$ dimensional nonnegative variable vector, $\mathbf{b}$ is an $m$-dimensional vector, $\mathbf{o}$ is an $n$-dimensional null-vector, and $*$ represents symbols $\leq,=$ and $\geq$.

Numerous computational methods are proposed for solving the model (1), but they are not efficient enough from the point of view of decision makers and analysts. Two important groups of methods are ones which use (a) interior point methods [10], and (b) simplex method after the linearization of the linear fractional objective functions. Here we investigate the efficiency of the linearization methods which use goal programming and the simplex method to solve the model (1).

\subsection{Solving the MOLFP model by goal programming}

To solve the model (1) by the goal programming method we have to find marginal solutions for all the objective functions on the given set of constraints with objective function values: $z_{1}^{*}, z_{2}^{*}, \ldots, z_{K}^{*}$. Later we form the goal programming model in one of the four possible ways ([15], [19], [20]):

(i) The $\min -\max$ form:

$\min _{n_{k}, p_{k}} \max _{(\mathbf{x}, \mathbf{n}, \mathbf{p}) \in \mathbf{S}_{\mathbf{1}}} g_{k}\left(n_{k}, p_{k}\right)$

where

$$
\mathbf{S}_{1}=\left\{\begin{array}{l}
(\mathbf{x}, \mathbf{n}, \mathbf{p}): \frac{c_{k} x+\alpha_{k}}{d_{k} x+\beta_{k}}+n_{k}-p_{k}=\bar{Z}_{k}, \\
k=1,2, \ldots, K, A \mathbf{x} * \mathbf{b}, \mathbf{x} \geq \mathbf{0}
\end{array}\right\}, n_{k} \geq 0, p_{k} \geq 0, n_{k} \cdot p_{k}=0,
$$


$k=1,2, \ldots, K$

Aspiration levels $\overline{z_{k}}$ are determined by the decision maker or equal to $z_{k}^{*} \cdot g_{k}$ is a linear function of the negative $\left(n_{k}\right)$ and positive $\left(p_{k}\right)$ deviational variables.

(ii) The minimization of the sum of deviations form:

$$
\min _{(\mathbf{x}, \mathbf{n}, \mathbf{p}) \in \mathbf{S}_{\mathbf{1}}} \sum_{k=1}^{K} g_{k}\left(n_{k}, p_{k}\right)
$$

(iii) The minimization of the weighted sum of deviations form:

$$
\min _{(\mathbf{x}, \mathbf{n}, \mathbf{p}) \in \mathbf{S}_{\mathbf{1}}} \sum_{k=1}^{K} w_{k} g_{k}\left(n_{k}, p_{k}\right)
$$

where $w_{k}(k=1,2, \ldots, K)$ are weights determined by the decision maker.

\section{(iv) The preemptive priority form:}

In this form the $K$ objectives are rearranged according to decision makers' priority levels, the highest priority goal is considered first, then the second and so on. The general lexicographical goal programme is:

$$
\min _{(\mathbf{x}, \mathbf{n}, \mathbf{p}) \in \mathbf{S}_{\mathbf{1}}}\left\{\sum_{k \in P_{i}} w_{k} g_{k}\left(n_{k}, p_{k}\right): i=1,2, \ldots, I\right\}
$$

where $I$ is the number of priority levels and $k \in P_{i}$ means that the $k$ th goal is at the $i$ th priority level.

Models (2), (3), (4) and (5) are nonlinear programming models which cannot be solved by the simplex method. The non-linear functions in the constraints set make a problem, which significantly complicates the solving process. To solve the models $(2-5)$ by the simplex method we have to apply a linearization technique to linearize the linear fractional objective functions. The complexity of the linearization approaches and problems with orthogonality constraints $n_{k} \cdot p_{k}=0$, $k=1,2, \ldots, K$ will be given in the rest of the paper. In this case we obtain an approximation of the model (2) that gives the week efficient solutions (see more in $[9,11,12,19,20])$.

\subsection{Taylor's polynomial linearization approximation}

The Taylor's polynomial linearization approximation is used to transform linear fractional functions into linear functions [22]. The fractional functions in the set of constraints of the specified goal programming models (2), (3), (4), and (5) are 
replaced by the linearized functions. In this way we obtain an appropriate linear programming model that can be solved by the simplex method.

The linearization procedure is performed in two steps:

Step 1) Determine $\mathbf{x}_{k}^{*}=\left[x_{k 1}^{*}, x_{k 2}^{*}, \ldots, x_{k n}^{*}\right]^{T}$ which is the value that maximizes the linear fractional objective function $z_{k}(x), k=1,2, \ldots, K$.

Step 2) Transform $z_{k}(x)$ by using the first-order Taylor polynomial series [22]. Consequently:

$$
z_{k}(\mathbf{x})=\frac{\mathbf{c}_{k} \mathbf{x}+c_{0}^{k}}{\mathbf{d}_{k} \mathbf{x}+d_{0}^{k}} \cong \hat{Z}_{k}(\mathbf{x})=z_{k}\left(\mathbf{x}_{k}^{*}\right)+\left[\left(x_{1}-x_{k 1}^{*}\right) \frac{\partial z_{k}\left(\mathbf{x}_{k}^{*}\right)}{\partial x_{1}}+\left(x_{2}-x_{k 2}^{*}\right) \frac{\partial z_{k}\left(\mathbf{x}_{k}^{*}\right)}{\partial x_{2}}+\ldots+\left(x_{n}-x_{k n}^{*}\right) \frac{\partial z_{k}\left(\mathbf{x}_{k}^{*}\right)}{\partial x_{n}}\right]
$$

Models (2), (3) and (4) obtained in this way are linear programming models that can be solved by the simplex method, and the model (5) can be solved by the multiphase simplex method [5] or the sequential simplex method [6]. Therefore, the linear goal programming model with the Taylor's polynomial linearization approach can be presented as

$$
\min _{(\mathbf{x}, \mathbf{d}) \in \mathbf{S}_{\mathbf{1}}} Z=\sum_{k=1}^{K} w_{k} g_{k}\left(d_{k}^{-}, d_{k}^{+}\right)
$$

where

$$
S_{1}=\left\{(\mathbf{x}, \mathbf{d}): \hat{z}_{k}+d_{k}^{-}-d_{k}^{+}=\overline{z_{k}}, A \mathbf{x} * b, \mathbf{x} \geq 0, d_{k}^{-}, d_{k}^{+} \geq 0, d_{k}^{-} \cdot d_{k}^{+}=0\right\}
$$

\subsection{Linearization using the method of variable change}

Pal, Moitra and Maulik [18] proposed the following linearization model for solving fuzzy MOLFP problems by goal programming methods:

Find $x$ so as to

$$
\min _{(\mathbf{x}, \mathbf{D}) \in \mathbf{S}_{2}} Z=\sum_{k=1}^{K} w_{k} g_{k}\left(D_{k}^{-}, D_{k}^{+}\right)
$$

where $\quad S_{2}=\left\{\begin{array}{l}(\mathbf{x}, \mathbf{D}): \mathbf{c}_{k} \mathbf{x}-\overline{z_{k}} \mathbf{d}_{k} \mathbf{x}+D_{k}^{-}-D_{k}^{+}=\overline{z_{k}} d_{0}^{k} c_{0}^{k}, \\ -z_{k}^{*} d_{k} \mathbf{x}+D_{k}^{-} \leq-z_{k}^{*} d_{0}^{k}, \\ A \mathbf{x}^{*} b, \mathbf{x} \geq 0, D_{k}^{-}, D_{k}^{+} \geq 0, D_{k}^{-} \cdot D_{k}^{+}=0\end{array}\right\}$,

$z$ represents the fully achievement function consisting of the weighted under and over-deviational variables, and numerical weights $w_{k}$ represent the relative importance of achieving the aspired levels of the respective fuzzy goals, 
$w_{k}^{-}=\frac{1}{\overline{Z_{k}}-l_{k}}$, (for functions to be maximized) or $w_{k}^{+}=\frac{1}{u_{k}-\overline{z_{k}}}$, (for functions to be minimized), $l_{k}$ is the lowest value of the $k$ th objective function, $u_{k}$ is the highest value of the $k$ th objective function, while $\overline{z_{k}}$ is the value determined by the decision maker.

\subsection{Linearization using a modification of the method of vari- able change}

To solve the multi-objective linear fractional programming model by the linear goal programming method for objective functions that are to be maximized or minimized, Perić and Babić [20] proposed the linearization of the linear fractional objective functions in the following way:

$$
\frac{\sum_{j=1}^{n} c_{k j} x_{j}+c_{0}^{k}}{\sum_{j=1}^{n} d_{k j} x_{j}+d_{0}^{k}} \leq(\geq) \overline{z_{k}} / \cdot\left(\sum_{j=1}^{n} d_{k j} x_{j}+d_{0}^{k}\right), \sum_{j=1}^{n} d_{k j} x_{j}+d_{0}^{k}>0
$$

for all $x_{j}, j=1,2, \ldots, n$,

$$
\begin{aligned}
& k=1,2, \ldots, K \Rightarrow \sum_{j=1}^{n} c_{k j} x_{j}+c_{0}^{k} \leq(\geq) \overline{z_{k}}\left(\sum_{j=1}^{n} d_{k j} x_{j}+d_{0}^{k}\right) \\
& \sum_{j=1}^{n}\left(c_{k j}-\overline{z_{k}} d_{k j}\right) x_{j} \leq \overline{z_{k}} d_{0}^{k}-c_{0}^{k} \Rightarrow \sum_{j=1}^{n} C_{k j} x_{j}+d_{k}^{-}-d_{k}^{+}=\overline{Z_{k}}, \quad k=1,2, \ldots, K,
\end{aligned}
$$

where $\overline{z_{k}}$ is the value of the $k$ th objective function determined by the decision maker, $C_{k j}=c_{k j}-\overline{z_{k}} d_{k j}, \overline{Z_{k}}=\overline{z_{k}} d_{0}^{k}-c_{0}^{k}, d_{k}^{-}$and $d_{k}^{+}$are under and overdeviational variables $(k=1,2, \ldots, K)$.

Therefore the goal programming model is presented as

$$
\min _{\mathbf{x} \in \mathbf{S}_{3}} g_{k}\left(d_{k}^{-}, d_{k}^{+}\right)
$$

where

$$
\mathbf{S}_{\mathbf{3}}=\left\{(\mathbf{x}, \mathbf{d}): C_{k j} x_{j}+d_{k}^{-}-d_{k}^{+}=\overline{Z_{k}}, k=1,2, \ldots, K, A \mathbf{x}^{*} b, \mathbf{x} \geq 0, d_{k}^{-}, d_{k}^{+} \geq 0, d_{k}^{-} \cdot d_{k}^{+}=0\right\}
$$


To solve the model (9) we can use numerous GP approaches for solving MOLGP models.

In multi-objective linear fractional programming problems, we often obtain weakly efficient ( $w$-efficient) solutions (points).

Below we recall the definitions of weakly efficient and efficient solutions of multiobjective programming problems. In a multi-objective programming problem with $k$ objective functions to be maximized, a decision vector $\mathbf{x}^{*} \in \mathbf{S}$ is weakly efficient (weakly Pareto optimal) if another decision vector $\mathbf{x} \in \mathbf{S}$ such that $f_{i}(\mathbf{x})>f_{i}\left(\mathbf{x}^{*}\right)$ for all $i=1,2, \ldots, k$ does not exist. In a multi-objective programming problem with $k$ objective functions to be maximized, a decision vector $\mathbf{x}^{*} \in \mathbf{S}$ is efficient (Pareto optimal) if another decision vector $\mathbf{x} \in \mathbf{S}$ such that $f_{i}(\mathbf{x}) \geq f_{i}\left(\mathbf{x}^{*}\right)$ for all $i=1,2, \ldots, k$ and $f_{j}(x)>f_{j}\left(x^{*}\right)$ for at least one index $j([13],[1])$ does not exist. Therefore, a Pareto optimal set is a subset of a weakly Pareto optimal set.

Since the set of all efficient points may not be entirely closed, this causes serious difficulties in trying to directly compute the set of all efficient points when the objective functions are linear fractional functions. Because the set of all w-efficient points is closed, it is easier to compute this set.

\section{Practical application: financial planning}

\subsection{A financial structure optimization problem}

Consider a firm which is expected to reach US $\$ 60.0$ million of capital in the next year. In order to increase the firm's value, the firm's financial manager wants to improve the financial condition of the company by constructing the optimal financial structure. Based on the expected sales for the next year, the aim is to maximize the manager's satisfaction with some financial ratios. Table 1 shows the variables which are considered. The four conflicting fractional goals are as follows: (1) minimization of current ratio, (2) minimization of debt ratio, (3) maximization of turnover ratio and (4) maximization of profitability ratio [19].

\begin{tabular}{|l|c|c|l|c|c|}
\hline Assets & Variable & $\begin{array}{c}\text { Expected } \\
\text { values in } \$ \\
\text { mill. }\end{array}$ & $\begin{array}{l}\text { Liabilities } \\
\text { and equity }\end{array}$ & Variable & $\begin{array}{c}\text { Expected } \\
\text { values }\end{array}$ \\
\hline $\begin{array}{l}\text { Current } \\
\text { assets }\end{array}$ & $x_{11}$ & $150 \leq x_{11} \leq 250$ & $\begin{array}{l}\text { Current } \\
\text { liabilities }\end{array}$ & $x_{21}$ & $75 \leq x_{21} \leq 300$ \\
\hline
\end{tabular}




\begin{tabular}{|l|c|c|l|c|c|}
\hline $\begin{array}{l}\text { Fixed } \\
\text { assets }\end{array}$ & $x_{12}$ & $x_{12} \leq 300$ & $\begin{array}{l}\text { Long-term } \\
\text { liabilities }\end{array}$ & $x_{22}$ & $x_{21}+x_{22} \geq 250$ \\
\hline $\begin{array}{l}\text { Total } \\
\text { assets }\end{array}$ & $x_{11}+x_{12}$ & $x_{11}+x_{12} \geq 350$ & $\begin{array}{l}\text { Shareholder } \\
\text { s equality }\end{array}$ & $x_{23}$ & $75 \leq x_{23} \leq 125$ \\
\hline Total & $x_{11}+x_{12}$ & $\begin{array}{l}\text { Retained } \\
\text { earnings } \\
\text { added }\end{array}$ & $x_{24}$ & $100 \leq x_{24} \leq 140$ \\
\hline & & $\begin{array}{l}\text { Total } \\
\text { liabilities } \\
\text { and } \\
\text { equality }\end{array}$ & $x_{21}+x_{22}+$ & $x_{23}+x_{24}$ & $x_{21}+x_{22}+x_{23}+x_{24}$ \\
\hline
\end{tabular}

Table 1: Definition of the variables in the balance sheet $(B / S)$

\subsection{MOLFP model}

The above data are the basis for the following MOLFP model:

$$
\max _{\mathbf{x} \in \mathbf{S}}\left\{z_{1}(\mathbf{x})=-\frac{x_{11}}{x_{21}}, z_{2}(\mathbf{x})=-\frac{x_{21}+x_{22}}{x_{23}+x_{24}}, z_{3}(\mathbf{x})=\frac{60}{x_{11}+x_{12}}, z_{4}(\mathbf{x})=\frac{x_{24}}{60}\right\},
$$

where

$\mathbf{S}=\left\{\begin{array}{l}\mathbf{x}: x_{11}+x_{12}=x_{21}+x_{22}+x_{23}+x_{24}, 150 \leq x_{11} \leq 250, x_{12} \leq 300, x_{11}+x_{12} \geq 350,75 \leq x_{21} \leq 300, \\ 100 \leq x_{22} \leq 300, x_{21}+x_{22} \geq 250,75 \leq x_{23} \leq 125,100 \leq x_{24} \leq 140, x_{11}, x_{12}, x_{21}, x_{22}, x_{23}, x_{24} \geq 0\end{array}\right\}$, $z_{1}, z_{2}, z_{3}$ and $z_{4}$ are functions of current ratio, debt ratio, turnover ratio and profitability ratio, respectively.

\subsection{The model solving}

Marginal solutions, obtained by maximizing each of the four objective functions individually on a given set of constraints using Charnes and Cooper's method [3], are presented in the following table: 


\begin{tabular}{|c|c|c|c|c|c|c|}
\hline Marginal & \multicolumn{2}{|c|}{ Variable values } & $z_{1}$ & $z_{2}$ & $z_{3}$ & $z_{4}$ \\
\hline \multirow{3}{*}{$x_{1}^{*}$} & $x_{11}=150$ & $x_{12}=300$ & \multirow[t]{3}{*}{-0.8571} & \multirow[t]{3}{*}{-1.5714} & \multirow[t]{3}{*}{0.1333} & \multirow[t]{3}{*}{1.6667} \\
\hline & $x_{21}=175$ & $x_{22}=100$ & & & & \\
\hline & $x_{23}=75$ & $x_{24}=100$ & & & & \\
\hline \multirow[t]{3}{*}{$x_{2}^{*}$} & $x_{11}=215$ & $x_{12}=300$ & \multirow[t]{3}{*}{-1.4333} & \multirow[t]{3}{*}{-0.9434} & \multirow[t]{3}{*}{0.1165} & \multirow[t]{3}{*}{2.3333} \\
\hline & $x_{21}=150$ & $x_{22}=100$ & & & & \\
\hline & $x_{23}=125$ & $x_{24}=140$ & & & & \\
\hline \multirow[t]{3}{*}{$x_{3}^{*}$} & $x_{11}=220$ & $x_{12}=205$ & \multirow[t]{3}{*}{-2.9333} & \multirow[t]{3}{*}{-1.4286} & \multirow[t]{3}{*}{0.1412} & \multirow[t]{3}{*}{1.6667} \\
\hline & $x_{21}=75$ & $x_{22}=175$ & & & & \\
\hline & $x_{23}=75$ & $x_{24}=100$ & & & & \\
\hline \multirow[t]{3}{*}{$x_{4}^{*}$} & $x_{11}=250$ & $x_{12}=300$ & \multirow[t]{3}{*}{-3.3333} & \multirow[t]{3}{*}{-1.5581} & \multirow[t]{3}{*}{0.1091} & \multirow[t]{3}{*}{2.3333} \\
\hline & $x_{21}=75$ & $x_{22}=260$ & & & & \\
\hline & $x_{23}=75$ & $x_{24}=140$ & & & & \\
\hline
\end{tabular}

Table 2: Marginal solutions

To solve the model by using presented linearization techniques we form the following goal programming models, which are solved using Excel Solver for Linear Programming:

Linearization model (1): The relation (6) is used to linearize the objective functions:

$$
\begin{aligned}
& z_{1}(\mathbf{x})=-\frac{x_{11}}{x_{21}} \cong z_{1}\left(\mathbf{x}_{1}^{*}\right)+\left(x_{11}-x_{11}^{*}\right) \frac{\partial z_{1}\left(\mathbf{x}_{1}^{*}\right)}{\partial x_{11}}+\left(x_{12}-x_{12}^{*}\right) \frac{\partial z_{1}\left(\mathbf{x}_{1}^{*}\right)}{\partial x_{12}}+\left(x_{21}-x_{21}^{*}\right) \frac{\partial z_{1}\left(\mathbf{x}_{1}^{*}\right)}{\partial x_{21}}+ \\
& +\left(x_{22}-x_{22}^{*}\right) \frac{\partial z_{1}\left(\mathbf{x}_{1}^{*}\right)}{\partial x_{22}}+\left(x_{23}-x_{23}^{*}\right) \frac{\partial z_{1}\left(\mathbf{x}_{1}^{*}\right)}{\partial x_{23}}+\left(x_{24}-x_{24}^{*}\right) \frac{\partial z_{1}\left(\mathbf{x}_{1}^{*}\right)}{\partial x_{24}}=-0.8571+ \\
& +\left(x_{11}-150\right) \cdot(-0.0057)+\left(x_{12}-300\right) \cdot 0+\left(x_{21}-175\right) \cdot 0.0049+\left(x_{22}-100\right) \cdot 0+ \\
& +\left(x_{23}-75\right) \cdot 0+\left(x_{24}-100\right) \cdot 0=-0.005714285 x_{11}+0.004897959 x_{21}-0.857142857
\end{aligned}
$$

The functions $z_{2}$ and $z_{3}$ are linearized analogously. Thus: 


$$
\begin{aligned}
z_{2}(\mathrm{x})=\frac{x_{21}+x_{22}}{x_{23}+x_{24}} \cong & -0.003773584 x_{21}-0.003773584 x_{22}+0.00359985 x_{23}+ \\
& +0.003559985 x_{24}+0.9434 \\
z_{3}(\mathbf{x})=\frac{60}{x_{11}+x_{12}} \cong & -0.000332179 x_{11}-0.000332179 x_{12}+0.282352575
\end{aligned}
$$

Based on this calculation we can form the linear goal programming model (iii) (the variant (a) when $\bar{Z}_{k}=z_{k}^{*}$, and the variant (b) when $z_{k}^{\min }<\bar{Z}_{k}<z_{k}^{*}$ ):

(a)

$$
\min _{(\mathbf{x}, \mathbf{n}) \in \mathbf{S}_{1}} \sum_{k=1}^{4} w_{k} n_{k}
$$

where

$$
\mathbf{S}_{1}=\left\{\begin{array}{l}
(\mathbf{x}, \mathbf{n}):-0.0057 x_{11}+0.0049 x_{21}-0.8571+n_{1}=\overline{z_{1}},-0.0038 x_{21}-0.0038 x_{22}+0.0036 x_{23}+ \\
0.0036 x_{24}-0.9434+n_{2}=\overline{z_{2}},-0.00033 x_{11}-0.00033 x_{12}+0.2824+n_{3}=\overline{z_{3}}, \frac{x_{24}}{60}+ \\
+n_{4}=\overline{z_{4}}, x_{11}+x_{12}=x_{21}+x_{22}+x_{23}+x_{24}, 150 \leq x_{11} \leq 250, x_{12} \leq 300, x_{11}+x_{12} \geq 350, \\
75 \leq x_{21} \leq 300,100 \leq x_{22} \leq 300, x_{21}+x_{22} \geq 250,75 \leq x_{23} \leq 125,100 \leq x_{24} \leq 140, \\
x_{11}, x_{12}, x_{21}, x_{22}, x_{23}, x_{24}, n_{1}, n_{2}, n_{3}, n_{4} \geq 0
\end{array}\right\},
$$

$\overline{z_{1}}=z_{1}^{*}=-0.8571, \quad \overline{z_{2}}=z_{2}^{*}=-0.9434, \quad \overline{z_{3}}=z_{3}^{*}=0.1412, \quad \overline{z_{4}}=z_{4}^{*}=2.3333$, $w_{1}=0.4038, w_{2}=1.5913, w_{3}=40.48$, and $w_{4}=1.5$. The weights $w_{1}, w_{2}, w_{3}$ and $w_{4}$ are calculated by $w_{k}=\frac{1}{u_{k}-\overline{z_{k}}}$, where $u_{k}$ is the biggest value of the $k$ th objective function on the given set.

$$
\min _{(\mathbf{x}, \mathbf{n}) \in \mathbf{S}_{\mathbf{1}}} \sum_{k=1}^{4} w_{k}\left(n_{k}+p_{k}\right)
$$

$$
\mathbf{S}_{1}{ }^{\prime}=\left\{\begin{array}{l}
(\mathbf{x}, \mathbf{n}, \mathbf{p}):-0.0057 x_{11}+0.0049 x_{21}-0.8571+n_{1}-p_{1}=\overline{z_{1}},-0.0038 x_{21}-0.0038 x_{22}+0.0036 x_{23}+ \\
0.0036 x_{24}-0.9434+n_{2}-p_{2}=\overline{z_{2}},-0.00033 x_{11}-0.00033 x_{12}+0.2824+n_{3}-p_{3}=\overline{z_{3}}, \frac{x_{24}}{60}+ \\
+n_{4}-p_{4}=\overline{z_{4}}, x_{11}+x_{12}=x_{21}+x_{22}+x_{23}+x_{24}, 150 \leq x_{11} \leq 250, x_{12} \leq 300, x_{11}+x_{12} \geq 350, \\
75 \leq x_{21} \leq 300,100 \leq x_{22} \leq 300, x_{21}+x_{22} \geq 250,75 \leq x_{23} \leq 125,100 \leq x_{24} \leq 140, \\
x_{11}, x_{12}, x_{21}, x_{22}, x_{23}, x_{24}, n_{1}, n_{2}, n_{3}, n_{4}, p_{1}, p_{2}, p_{3}, p_{4} \geq 0, n_{k} \cdot p_{k}=0, k \in\{1,2,3,4\}
\end{array}\right\},
$$


$\overline{z_{1}}=-1.15, \quad \overline{z_{2}}=-1.25, \quad \overline{z_{3}}=0.12, \quad \overline{z_{4}}=1.85, \quad w_{1}=3.4141, \quad w_{2}=3.2616$, $w_{3}=47.1698$, and $w_{4}=20.6911$. In the variant (b) $\overline{z_{1}}, \overline{z_{2}}, \overline{z_{3}}, \overline{z_{4}}$ are given by the decision maker, while $\boldsymbol{w}_{1}, w_{2}, w_{3}$, and $w_{4}$ are calculated by $w_{k}=\frac{1}{u_{k}-\overline{z_{k}}}$, where $u_{k}$ is the biggest value of the $k$ th objective function.

Linearization model (2): Using the relation (8) we solve the following models:

$$
\min _{(\mathbf{x}, \mathbf{D}) \in \mathbf{S}_{2}} \sum_{k=1}^{w} w_{k} D_{k}^{-},
$$

where

$\mathbf{S}_{2}=\left\{\begin{array}{l}(\mathbf{x}, \mathbf{D}):-x_{11}+0.8571 x_{21}+D_{1}^{-}=0,-0.8571 x_{21}+D_{1}^{-} \leq 0,-x_{21}-x_{22}+0.9434 x_{23}+0.9434 x_{24}+ \\ +D_{2}^{-}=0,-0.9434 x_{23}-0.9434 x_{24}+D_{2}^{-} \leq 0,-0.1412 x_{11}-0.1412 x_{12}+D_{3}^{-}=-60, \\ -0.1412 x_{11}-0.1412 x_{12}+D_{3}^{-} \leq 0, \frac{x_{24}}{60}+D_{4}^{-}=2.3333, x_{24}+60 D_{4}^{-} \leq 0, x_{11}+x_{12}=x_{21}+x_{22}+ \\ +x_{23}+x_{24}, 150 \leq x_{11} \leq 250, x_{12} \leq 300, x_{11}+x_{12} \geq 350,75 \leq x_{21} \leq 300,100 \leq x_{22} \leq 300, x_{21}+x_{22} \geq 250, \\ 75 \leq x_{23} \leq 125,100 \leq x_{24} \leq 140, x_{11}, x_{12}, x_{21}, x_{22}, x_{23}, x_{24}, D_{1}^{-}, D_{2}^{-}, D_{3}^{-}, D_{4}^{-} \geq 0\end{array}\right\}$,

$\overline{z_{1}}=z_{1}^{*}=-0.8571, \quad \overline{z_{2}}=z_{2}^{*}=-0.9434, \quad \overline{z_{3}}=z_{3}^{*}=0.1412, \quad \overline{z_{4}}=z_{4}^{*}=2.3333$, $w_{1}=0.4038, w_{2}=1.5913, w_{3}=40.48$, and $w_{4}=1.5$.

$$
\min _{(\mathbf{x}, \mathbf{D}) \in \mathbf{S}_{\mathbf{2}}} \sum_{k=1}^{w} w_{k}\left(D_{k}^{-}+D_{k}^{+}\right)
$$

where

$$
\begin{aligned}
& \mathbf{S}_{2}{ }^{\prime}=\left\{\begin{array}{l}
(\mathbf{x}, \mathbf{D}):-x_{11}+1.15 x_{21}+D_{1}^{-}-D_{1}^{+}=0,-0.8571 x_{21}+D_{1}^{-} \leq 0,-x_{21}-x_{22}+1.25 x_{23}+1.25 x_{24}+ \\
+D_{2}^{-}-D_{2}^{+}=0,-0.9434 x_{23}-0.9434 x_{24}+D_{2}^{-} \leq 0,-0.12 x_{11}-0.12 x_{12}+D_{3}^{-}-D_{3}^{+}=-60, \\
-0.1412 x_{11}-0.1412 x_{12}+D_{3}^{-} \leq 0, \frac{x_{24}}{60}+D_{4}^{-}-D_{4}^{+}=1.85, x_{24}+60 D_{4}^{-} \leq 0, x_{11}+x_{12}=x_{21}+x_{22}+ \\
+x_{23}+x_{24}, 150 \leq x_{11} \leq 250, x_{12} \leq 300, x_{11}+x_{12} \geq 350,75 \leq x_{21} \leq 300,100 \leq x_{22} \leq 300, \\
x_{21}+x_{22} \geq 250,75 \leq x_{23} \leq 125,100 \leq x_{24} \leq 140, x_{11}, x_{12}, x_{21}, x_{22}, x_{23}, x_{24}, D_{1}^{-}, D_{2}^{-}, D_{3}^{-}, D_{4}^{-}, \\
D_{1}^{+}, D_{2}^{+}, D_{3}^{+}, D_{4}^{+} \geq 0, D_{k}^{-} \cdot D_{k}^{+}=0, k \in\{1,2,3,4\}
\end{array}\right\}, \\
& \overline{z_{1}}=-1.15, \overline{z_{2}}=-1.25, \overline{z_{3}}=0.12, \overline{z_{4}}=1.85, w_{1}=3.4141, w_{2}=3.2616, \\
& w_{3}=47.1698, \text { and } w_{4}=20.6911 .
\end{aligned}
$$

Linearization model (3): Using the relation (8) we solve the following models:

$$
\min _{(\mathbf{x}, \mathbf{d}) \in \mathbf{S}_{3}} \sum_{k=1}^{4} w_{k} d_{k}^{-},
$$


where

$$
\mathbf{S}_{3}=\left\{\begin{array}{l}
(\mathbf{x}, \mathbf{d}):-x_{11}+0.8571 x_{21}+d_{1}^{-}=0,-x_{21}-x_{22}+0.9434 x_{23}+0.9434 x_{24}+d_{2}^{-}=0, \\
-0.1412 x_{11}-0.1412 x_{12}+d_{3}^{-}=-60, \frac{x_{24}}{60}+d_{4}^{-}=2.3333 \\
x_{11}+x_{12}=x_{21}+x_{22}+x_{23}+x_{24}, 150 \leq x_{11} \leq 250, x_{12} \leq 300, x_{11}+x_{12} \geq 350, \\
75 \leq x_{21} \leq 300,100 \leq x_{22} \leq 300, x_{21}+x_{22} \geq 250,75 \leq x_{23} \leq 125,100 \leq x_{24} \leq 140, \\
x_{11}, x_{12}, x_{21}, x_{22}, x_{23}, x_{24}, d_{1}^{-}, d_{2}^{-}, d_{3}^{-}, d_{4}^{-} \geq 0
\end{array}\right\},
$$

$\overline{z_{1}}=z_{1}^{*}=-0.8571, \overline{z_{2}}=z_{2}^{*}=-0.9434, \overline{z_{3}}=z_{3}^{*}=0.1412, \overline{z_{4}}=z_{4}^{*}=2.3333$,

$w_{1}=0.4038, w_{2}=1.5913, w_{3}=40.48$, and $w_{4}=1.5$.

(b)

$$
\min _{(\mathbf{x}, \mathbf{d}) \in \mathbf{S}_{3}} \sum_{k=1}^{4} w_{k}\left(d_{k}^{-}+d_{k}^{+}\right)
$$

where

$$
\mathbf{S}_{3}{ }^{\prime}=\left\{\begin{array}{l}
(\mathbf{x}, \mathbf{d}):-x_{11}+1.15 x_{21}+d_{1}^{-}-d_{1}^{+}=0,-x_{21}-x_{22}+1.25 x_{23}+1.25 x_{24}+d_{2}^{-}-d_{2}^{+}=0, \\
-0.12 x_{11}-0.12 x_{12}+d_{3}^{-}-d_{3}^{+}=-60, \frac{x_{24}}{60}+d_{4}^{-}-d_{4}^{+}=1.85, \\
x_{11}+x_{12}=x_{21}+x_{22}+x_{23}+x_{24}, 150 \leq x_{11} \leq 250, x_{12} \leq 300, x_{11}+x_{12} \geq 350, \\
75 \leq x_{21} \leq 300,100 \leq x_{22} \leq 300, x_{21}+x_{22} \geq 250,75 \leq x_{23} \leq 125,100 \leq x_{24} \leq 140, \\
x_{11}, x_{12}, x_{21}, x_{22}, x_{23}, x_{24}, d_{1}^{-}, d_{2}^{-}, d_{3}^{-}, d_{4}^{-}, d_{1}^{+}, d_{2}^{+}, d_{3}^{+}, d_{4}^{+} \geq 0, d_{k}^{-} \cdot d_{k}^{+}=0, k \in\{1,2,3,4\}
\end{array}\right\},
$$

$\overline{z_{1}}=-1.15, \overline{z_{2}}=-1.25, \overline{z_{3}}=0.12, \overline{z_{4}}=1.85, \quad w_{1}=3.4141, \quad w_{2}=3.2616$, $w_{3}=47.1698$, and $w_{4}=20.6911$.

\subsection{The solutions}

The solutions of the models (11), (12), and (16) are given in Tables 3 and 4.

\begin{tabular}{|l|c|c|c|c|}
\hline \multicolumn{1}{|c|}{ GP solution } & $z_{1}$ & $z_{2}$ & $z_{3}$ & $z_{4}$ \\
\hline $\begin{array}{l}\text { Linearization } \\
\text { model }(1)\end{array}$ & -1.09812 & -1.16431 & 0.12911 & 2.32864 \\
\hline $\begin{array}{l}\text { Linearization } \\
\text { model (2) }\end{array}$ & -1.00 & -0.14286 & 0.14118 & 1.66667 \\
\hline $\begin{array}{l}\text { Linearization } \\
\text { model (3) }\end{array}$ & -1.00 & -0.14286 & 0.14118 & 1.66667 \\
\hline
\end{tabular}

Table 3: Goal programming solutions, version (a) 


\begin{tabular}{|l|c|c|c|c|}
\hline \multicolumn{1}{|c|}{ GP solution } & $z_{1}$ & $z_{2}$ & $z_{3}$ & $z_{4}$ \\
\hline $\begin{array}{l}\text { Linearization } \\
\text { model (1) }\end{array}$ & -1.20222 & -1.16431 & 0.12911 & 2.32864 \\
\hline $\begin{array}{l}\text { Linearization } \\
\text { model (2) }\end{array}$ & -1.00 & -0.14286 & 0.14118 & 1.66667 \\
\hline $\begin{array}{l}\text { Linearization } \\
\text { model (3) }\end{array}$ & -1.00 & -0.14286 & 0.14118 & 1.66667 \\
\hline
\end{tabular}

Table 4: Goal programming solutions, version (b)

Tables 3 and 4 show that the solutions of the problem solved by model (1) are different from the solutions obtained by models (2) and (3) in both versions. It should be noted that the objective function weights in versions (a) and (b) are different. Also, changing the weights of the objective functions by solving the linearization models (1), (2) and (3) in both variants, we have concluded that the model (1) is not sensitive to the changes of the objective function weights in both variants. The linearization models (2) and (3) in our example give the same solution. It is because the linearization models (2) and (3) differ only in the fact that model (2) contains the additional constraints

$$
\sum_{j=1}^{K} d_{k j} x_{j}+d_{0}^{k}>0
$$

which are in our example proven to be redundant. Solving the models (2) and (3) in both variants by varying the objective function weights, we obtain the weak efficient solutions which reflect the preferences of the decision maker, and greatly improve the process of problem solving.

The model (3) is the most applicable from the point of view of analysts. Here we solve a linear programming problem where the additional constraints are constituted by the linearized objective functions. The linearization process is not demanding for the analyst. Model (2) gives the same solutions as the model (3), but it requests forming two additional constraints for each objective function. The model (1) requests the linearization of the objective functions by Taylor's polynomial approximation, which requires an additional effort and increases the possibility of errors, and in our example is not sensitive to the changes of the objective function weights as well. The sensitivity of the model (1) to changes of the objective function weights should be investigated theoretically and by solving more different practical multi-objective linear fractional programming problems. According to the brief discussion given above we conclude that the model (3) is the most applicable in both variants for solving the problem of the financial structure optimization by goal programming.

At the end of the discussion it should be noted that the accuracy of the linearized models has been investigated by comparing the marginal solutions obtained by 
using Charnes and Cooper's method and the linearized linear programming models in solving our problem whereby we got the same solutions when we used the linearization models (2) and (3). Small differences in the marginal values of the objective function appeared in the application of linearization model (1). The linearization model (3) is computationally simple where it is a good approximation of an exact method. How much it outperforms an exact MOLFP method it should be further investigated on more examples with a big number linear fractional objective functions, variables and constraints. Also theoretically the efficiency of the obtained solutions should be investigated.

\section{Concluding remarks}

This paper presents three different approaches to the linearization of linear fractional functions for solving multi objective linear fractional programming problems by using goal programming methodology.

The presented methodologies are tested on the example of a financial structure optimization problem in a company. The analysis of applicability of the linearization methodologies indicates the most appropriate linearization approach for solving the financial structure optimization problem is linearization model (3). The use of the chosen methodology is simple for both the analyst and the decision maker. If the decision maker determines objective function weights, then the obtained solutions reflect the decision maker's preferences. The obtained solutions are weakly efficient.

For future research, we suggest testing the computational characteristics of the linearization approach using the examples with a large number of objective functions, variables and constraints and theoretically testing the efficiency of the obtained solutions.

\section{Acknowledgement}

This work has been fully supported by the Croatian Science Foundation under the project STRENGTHS No 9402. We are also thankful to anonymous reviewers for their useful suggestions.

\section{References}

[1] Caballero, R. and Hernandez, M. (2006). Restoration of efficiency in a goal programming problem with linear fractional criteria. European Journal of Operational Research, 172, 31-39. 
[2] Chakraborty, M. and Gupta, S. (2002) Fuzzy mathematical programming for multi objective linear fractional programming problem. Fuzzy Sets and Systems, 125, 335-342.

[3] Charnes, A. and Cooper, W.W. (1961). Management Models of Industrial Applications of Linear Programme (Appendix B), vol. -1, Wiley, New York.

[4] Craven, B.D. (1988). Fractional Programming, Heldermann Verlag, Berlin.

[5] Hannan, E.L. (1981). Linear programming with multiple fuzzy goals. Fuzzy sets and systems, 6, 235-248.

[6] Ignizio, J.P. (1976). Goal Programming and Extensions, Lexington D.C.Health. MA.

[7] Kao, C. and Liu, S.T. (2001). Fractional programming approach to fuzzy weighted average, Fuzzy Sets and Systems, 120, 435-444.

[8] Kornbluth, J.S.H. and Steuer, R.E. (1981). Goal programming with linear fractional criteria, European Journal of Operational Research, 8, 58-65.

[9] Kornbluth, J.S.H. and Steuer, R.E. (1981). Multiple objective linear fractional programming, Management Science, 27, 1024-1039.

[10] Lesaja, G. (2009). Introducing interior-point methods for introductory operations research courses and/or linear programming courses. The Open Operational Research Journal, 3, 1-12.

[11] Mehrjerdi, Y.Z. (2011). Solving fractional programming problem through fuzzy goal setting and approximation. Applied Soft Computing, 11, 17351742.

[12] Metev, B. and Gueorguieva, D. (2000). A simple method for obtaining weakly efficient points in multiobjective linear fractional programming problems. European Journal of Operational Research, 126, 386-390.

[13] Miettinen, M. K., (1998) Nonlinear multiobjective optimization. Kluwer Academic Publishers, Norwell, USA.

[14] Mishra, B. and Singh, S.R. (2013). Linear fractional programming procedure for multiobjective linear programming problem in agricultural system. International Journal of Computer Applications, 60, 0975-8887.

[15] Mohamed, R.H. (1997). The relationship between goal programming and fuzzy programming. Fuzzy sets and systems, 89, 215-222.

[16] Ohta, H. and Yamaguchi, T. (1996). Linear fractional goal programming in consideration of fuzzy solution. European Journal of Operational Research 92, $157-165$.

[17] Pal, B.B. and Basu, I. (1995). A goal programming method for solving fractional programming problems via dynamic programming. Optimization, $35,145-157$.

[18] Pal, B.B., Moitra, B.N., Maulik, U. (2003) A goal programming procedure for fuzzy multiobjective linear fractional programming problem. Fuzzy Sets and Systems, 139, 395-405. 
[19] Perić, T. and Babić, Z. (2012) Financial structure optimization by using a goal programming approach. Croatian Operational Research Review, 3, 150162.

[20] Perić, T., Babić, Z., A new goal programming approach for multiobjective fractional linear programming. Proceedings of the 25th DAAAM International Symposium on Inteligent Manufacturing and Automation, DAAAM 2014, Ed. B. Katalinić, Vienna, November 26-29, 2014, CDROM Version.

[21] Sakawa, M. and Yumine, T. (1983). Interactive fuzzy decision making for multiobjective linear fractional programming problems. Large Scale Systems, $5,105-114$.

[22] Toksari, D.M. (2008), Taylor series approach to fuzzy multiobjective linear fractional programming. Informational Sciences 178, 1189-1204. 